Bull. Austral. Math. Soc.

$46 \mathrm{~B} 20,47 \mathrm{H} 05,41 \mathrm{~A} 65$

VoL. 58 (1998) [75-92]

\title{
FARTHEST POINTS AND MONOTONE OPERATORS
}

\section{U. Westphal AND T. SchWaRTZ}

Dedicated to $\mathrm{H}$. Berens on the occasion of his sixtieth birthday

\begin{abstract}
We apply the theory of monotone operators to study farthest points in closed bounded subsets of real Banach spaces. This new approach reveals the intimate connection between the farthest point mapping and the subdifferential of the farthest distance function. Moreover, we prove that a typical exception set in the Baire category sense is pathwise connected. Stronger results are obtained in Hilbert spaces.
\end{abstract}

\section{INTRODUCTION}

Let $X$ be a real Banach space with norm $\|\cdot\|$ and dual space $X^{*}$, and let $K$ be a nonempty closed bounded subset of $X$. The farthest distance function $r_{K}: X \rightarrow \mathbb{R}$ associated with $K$ is defined by

$$
r_{K}(x)=\sup \{\|x-k\| ; k \in K\} \quad(x \in X) .
$$

An element $k \in K$ is said to be a farthest point of $x \in X$, if $\|x-k\|=r_{K}(x)$. This gives rise to a set-valued mapping $Q_{K}: X \rightarrow 2^{K}$ defined by

$$
Q_{K}(x)=\left\{k \in K ;\|x-k\|=r_{K}(x)\right\} \quad(x \in X),
$$

which is called the farthest point mapping from $X$ to $K$. A simple observation shows that each point in the domain of $Q_{K}$ lies on a ray all points of which have a common farthest point. We call this phenomenon the "ray property" of $Q_{K}$ (see Section 3).

The purpose of this paper is to study farthest points by monotonicity methods. Indeed, as the farthest distance function $r_{K}$ is convex and continuous, its subdifferential $\partial r_{K}$ is a maximal monotone operator from $X$ to $X^{*}$. It extends the monotone operator $F \cdot \frac{I-Q_{K}}{r_{K}}$, where $F$ means the duality mapping of $X$ and $\frac{I-Q_{K}}{r_{K}}$ is the operator of "ray directions" related to the ray property of $Q_{K}$. If $X$ is even a Hilbert space, a further monotone operator is available: The negative of the farthest point mapping, $-Q_{K}$, is then monotone and has a unique maximal monotone extension, which is the subdifferential of a continuous convex function. The latter has a counterpart for nearest points in Hilbert

Received 26th November, 1998

Copyright Clearance Centre, Inc. Serial-fee code: 0004-9729/98 $\$ A 2.00+0.00$. 
spaces. Originating from Asplund [2], several papers of Berens-Westphal [9], Berens [8], Westphal-Frerking [25], and Veselý [24] considered the aspect of monotony of the metric projection and its relations to best approximation in Hilbert spaces in some detail. These papers were stimulating for the present one. However, our results go definitely beyond mere analogues. In contrast to the situation of best approximation, it is possible in the context of farthest points to apply the theory of monotone operators also in nonHilbert spaces owing to the convexity of $r_{K}$ in an arbitrary Banach space. Thus, it is the subdifferential $\partial r_{K}$ which is the main object of investigations in this paper.

Before describing the content of each section let us introduce some notation.

The open ball, closed ball, and sphere with centre $x \in X$ and radius $r>0$ will be denoted by $B(x ; r), \bar{B}(x ; r)$, and $S(x ; r)$, respectively. The symbols $B^{*}\left(x^{*} ; r\right), \bar{B}^{*}\left(x^{*} ; r\right)$, and $S^{*}\left(x^{*} ; r\right)$ stand for the corresponding subsets of $X^{*}$. The closed convex hull of a set $M \subset X$ will be denoted by $\overline{c o} M$, its cardinality by $|M|$.

The paper is organised as follows. In Section 2 we review some basic facts on monotone operators. Section 3 is concerned with the farthest point mapping, especially with its ray property. In Sections 4 and 5 we study the subdifferential of $r_{K}$. We start with a result on its range sharpening the well-known fact that all subgradients of $r_{K}$ are contained in the closed unit ball of $X^{*}$. Then the relationship of the subdifferential $\partial r_{K}$ to the farthest point mapping is analysed. For this, we distinguish between those subgradients of $r_{K}$ lying on the boundary and those contained in the interior of the unit ball of $X^{*}$. Among others, we show that if $X$ is a reflexive Banach space satisfying certain geometrical properties, then

$$
\partial r_{K}(x) \cap S^{*}(0 ; 1)=F\left(\frac{x-Q_{\overline{\mathrm{co}} K}(x)}{r_{K}(x)}\right) \quad(x \in X),
$$

which implies that the inverse image of a subgradient of norm one is actually a ray. In Section 5 we apply the resolvent theory for monotone operators to prove that in each reflexive Banach space $X$ the set

$$
\left\{x \in X ; \partial r_{K}(x) \cap B^{*}(0 ; 1) \neq \emptyset\right\}
$$

which is a typical exception set in the Baire category sense, is pathwise connected. This generalises a result of Balaganskiî [3] on the set of discontinuity points of the farthest point mapping. In Section 6 we obtain stronger results in Hilbert spaces involving the monotony of $-Q_{K}$.

\section{MONOTONE OPERATORS}

In this section we recall some basic results on monotone operators; for details we refer to $[6,11,14,26]$. Some of the statements below, such as Proposition 2.1 and formula (2.1), are modelled for our purposes. 
Let $X$ be a real Banach space. The symbol $\langle\cdot, \cdot\rangle$ will denote the canonical bilinear form on $X \times X^{*}$. If $X$ is a Hilbert space, it will be identified with its dual, and then $\langle\cdot, \cdot\rangle$ stands for the inner product of $X$.

Let us first recall the duality mapping of $X$ which is actually an example of a maximal monotone operator. It is the set-valued mapping $F: X \rightarrow 2^{X^{*}}$ defined by

$$
F(x)=\left\{x^{*} \in X^{*} ;\left\langle x, x^{*}\right\rangle=\|x\|^{2}=\left\|x^{*}\right\|^{2}\right\} \quad(x \in X) .
$$

$F$ is surjective if and only if $X$ is reflexive; in this case we identify the duality mapping of $X^{*}$ with the inverse $F^{-1}$ of $F$. The duality mapping reflects the metric geometry of the underlying space; see for example, the book of Cioranescu [12]. Indeed, $X$ is smooth [strictly convex] if and only if $F$ is single-valued [injective]. Moreover, the norm of $X$ is Fréchet differentiable on $X \backslash\{0\}$ if and only if $F$ is single-valued and norm to norm continuous. Note that the dual norm is Fréchet differentiable at each nonzero point of $X^{*}$ exactly when $X$ is reflexive, strictly convex, and satisfies the Kadec property which means that for sequences in the unit sphere weak and norm convergence agree. Such a space has been called "strongly convex" in the Russian literature.

A set-valued mapping $A: X \rightarrow 2^{X^{*}}$ with domain $D(A)$ and range $R(A)$ is usually identified with its graph in $X \times X^{*}$. It is said to be monotone, if

$$
\left\langle x-y, x^{*}-y^{*}\right\rangle \geqslant 0 \quad \forall\left(x, x^{*}\right),\left(y, y^{*}\right) \in A .
$$

A monotone mapping is called maximal monotone, if it has no proper monotone extension in $X \times X^{*}$.

Let $A$ be maximal monotone. Then it is demiclosed, that is, if $\left(x_{n}, x_{n}^{*}\right) \in A$ such that $x_{n} \rightarrow x$ and $x_{n}^{*} \rightarrow x^{*}$ (in the weak* topology of $X^{*}$ ), then $\left(x, x^{*}\right) \in A$. Moreover, for each $x \in D(A), A x$ is a convex and weak* closed subset of $X^{*}$. Finally, $A$ is norm to weak $^{*}$ upper semicontinuous on the interior of its domain.

Now, let $X$ be a reflexive space. Then a mapping $A: X \rightarrow 2^{X^{*}}$ is maximal monotone if and only if its inverse $A^{-1}: X^{*} \rightarrow 2^{X}$ is so. In this case, $\overline{D(A)}$ and $\overline{R(A)}$ are convex subsets of $X$ and $X^{*}$, respectively. Moreover, every monotone operator $B: X \rightarrow 2^{X^{*}}$ has a maximal monotone extension $A$ such that $\overline{D(A)}=\overline{c o} D(B)$. If $B$ is densely defined on $X$ and the domain of $A$ is all of $X$, then one even gets uniqueness of the maximal monotone extension. For later reference we state the following proposition.

Proposition 2.1. Let $X$ be a reflexive Banach space, and let $B: X \rightarrow 2^{X^{*}}$ be a monotone operator such that $\overline{D(B)}=X$. If there exists a maximal monotone extension $A$ of $B$ such that $D(A)=X$, then, for each $x \in X$, the set $A x$ can be represented by

$$
A x=\bigcap_{\delta>0} \overline{c o}\{B y ;\|y-x\|<\delta\}
$$

and $A$ is the unique maximal monotone extension of $B$. Moreover, $\overline{R(A)}=\overline{\operatorname{co}} R(B)$. 
Now, in addition to reflexivity, suppose that $X$ is strictly convex and smooth. If $A: X \rightarrow 2^{X^{*}}$ is a maximal monotone operator, then, for each positive number $\lambda$, its resolvent

$$
J_{\lambda}:=\left(I+\lambda F^{-1} \cdot A\right)^{-1}
$$

as well as its Yosida approximation

$$
A_{\lambda}:=F \cdot \frac{I-J_{\lambda}}{\lambda}
$$

are single-valued operators and defined on all of $X$. The range of $J_{\lambda}$ is $D(A)$, and

$$
\left(J_{\lambda} x, A_{\lambda} x\right) \in A \quad \forall x \in X .
$$

Moreover, for each $x \in \overline{D(A)}$,

$$
\lim _{(y, \lambda) \rightarrow(x, 0+)} J_{\lambda} y=x
$$

Concerning continuity, the resolvent $J_{\lambda}: X \rightarrow D(A)$ is norm to weak continuous, and if, in addition, the norm of $X^{*}$ is Fréchet differentiable, then $J_{\lambda}$ is even norm to norm continuous. The Yosida approximation $A_{\lambda}: X \rightarrow X^{*}$ is a maximal monotone mapping and hence norm to weak* continuous; if, in addition, the norm of $X$ is Fréchet differentiable, then $A_{\lambda}$ is continuous with respect to the norm topology of both $X$ and $X^{*}$.

If $X$ is a Hilbert space, maximal monotone operators are characterised by Minty's theorem which says that an operator $A: X \rightarrow 2^{X}$ is maximal monotone if and only if, for each $\lambda>0$, the resolvent $J_{\lambda}=(I+\lambda A)^{-1}$ is a contraction defined on all of $X$.

An important class of maximal monotone operators is given by the subdifferentials of proper, lower semicontinuous, convex functions. If $\varphi: X \rightarrow(-\infty, \infty)$ is such a function, then its subdifferential at $x \in X$ is defined by

$$
\partial \varphi(x):=\left\{x^{*} \in X^{*} ; \varphi(x)+\left\langle y-x, x^{*}\right\rangle \leqslant \varphi(y) \quad \forall y \in X\right\}
$$

the elements $x^{*} \in \partial \varphi(x)$ being called the subgradients of $\varphi$ at $x$. In this paper we are concerned with real-valued convex functions which are continuous on the whole space $X$. In this case, the domain $D(\partial \varphi)=\{x \in X ; \partial \varphi(x) \neq \emptyset\}$ is all of $X$.

Note that the subdifferential of the function $x \mapsto\|x\|^{2} / 2$ is just the duality mapping of $X$.

\section{The FARTHEST POINT MAPPING $Q_{K}$}

This section contains some basic facts on the farthest point mapping $Q_{K}$ which will be used in the sequel. In particular, we discuss what we call the "ray property" of $Q_{K}$ as far as it is important for investigating the subdifferential of the farthest distance function $r_{K}$. 
Suppose $K$ is a nonempty closed bounded subset of a Banach space $X$. Obviously, the farthest distance function of $K$ and its closed convex hull always coincide, that is

$$
r_{\overline{c o} K}=r_{K}
$$

The corresponding result for the farthest point mapping is true under assumptions which guarantee that every farthest point in $\overline{c o} K$ is a strongly exposed point of $\overline{c o} K$ (see $[17,21])$. For instance, if the norm of $X^{*}$ is Fréchet differentiable, then

$$
Q_{\overline{\mathrm{co}} K}=Q_{K}
$$

Extending results of Edelstein [16] and Asplund [1], Lau [21] showed that for any weakly compact subset $K$ of an arbitrary Banach space $X$ the domain of $Q_{K}$ contains a dense $G_{\delta}$ set of $X$. This implies that in a reflexive space $Q_{\overline{c o} K}$ is densely defined on $X$. Moreover, if the norm of $X^{*}$ is Fréchet differentiable, then by $(3.1) \overline{D\left(Q_{K}\right)}=X$. For further results on generic existence of farthest points see [15, 27].

Concerning the range of $Q_{K}$, one has

$$
\overline{\mathrm{co}} R\left(Q_{K}\right)=\overline{\mathrm{co}} K,
$$

if the norms of both $X$ and $X^{*}$ are Fréchet differentiable. This result goes back to Edelstein [16]; see also Lau [21].

Continuity and single-valuedness of the farthest point mapping were studied for example, by Blatter [10] and Zhivkov [27, 28]; for a systematic discussion of this subject and its relation to differentiability of the farthest distance function see Fitzpatrick [18].

We now turn to the "ray property" of $Q_{K}$. For this, suppose that $|K| \geqslant 2$. If $x \in D\left(Q_{K}\right)$ and $k \in Q_{K}(x)$, set $u:=\frac{x-k}{r_{K}(x)}$. Obviously, $k$ is also a farthest point of all points on the ray originating from $x$ and running in the direction of $u$, that is, $k \in Q_{K}(k+t u)$ for each $t \geqslant r_{K}(x)$. Let

$$
t_{0}:=\inf \left\{t>0 ; k \in Q_{K}(k+t u)\right\} .
$$

As $|K| \geqslant 2, t_{0}$ is a positive number and even a minimum. Hence, given $k \in R\left(Q_{K}\right)$, the set $Q_{K}{ }^{-1}(k)$ of all elements having $k$ as a farthest point is the union of pairwise disjoint rays of the form

$$
\left\{k+t u ; t \geqslant t_{u}\right\},
$$

where $u$ is a unit vector indicating the direction of the ray and $t_{u}$ is a positive real number. The set of all ray directions $u$ arising in this way from the elements of $R\left(Q_{K}\right)$ is the range of the set-valued mapping $\frac{I-Q_{K}}{r_{K}}$, which assigns to each $x \in X$ the set $\frac{x-Q_{K}(x)}{r_{K}(x)}$. The following proposition states that if $X$ is strictly convex, then any two rays in $D\left(Q_{K}\right)$ of the form (3.3) fail to be parallel. 
PROPOSITION 3.1. Let $X$ be a strictly convex Banach space. If $u \in R\left(\frac{I-Q_{K}}{r_{K}}\right)$, then there exists a unique element $k \in K$ such that $k \in Q_{K}(k+t u)$ for all sufficiently large $t$. Furthermore,

$$
\left(\frac{I-Q_{K}}{r_{K}}\right)^{-1}(u)=\left\{k+t u ; t \geqslant t_{0}\right\}
$$

where $t_{0}:=\min \left\{t>0 ; k \in Q_{K}(k+t u)\right\}$. If $t>t_{0}$, then $k=Q_{K}(k+t u)$.

Proof: By assumption, there exists $(x, k) \in Q_{K}$ such that $u=\frac{x-k}{r_{K}(x)}$. Set $t_{0}:=$ $\min \left\{t>0 ; k \in Q_{K}(k+t u)\right\}$ and $y_{t}:=k+t u$ for $t \geqslant t_{0}$. We know that $k \in Q_{K}\left(y_{t}\right)$ for all $t \geqslant t_{0}$ and thus

$$
\left\{y_{t} ; t \geqslant t_{0}\right\} \subset\left(\frac{I-Q_{K}}{r_{K}}\right)^{-1}(u) .
$$

Now let $t>t_{0}$ and $k^{\prime} \in Q_{K}\left(y_{t}\right)$. Then

$$
\begin{aligned}
r_{K}\left(y_{t}\right) & =\left\|y_{t_{0}}-k^{\prime}+\left(t-t_{0}\right) u\right\| \\
& \leqslant\left\|y_{t_{0}}-k^{\prime}\right\|+\left(t-t_{0}\right)\|u\| \\
& \leqslant\left\|y_{t_{0}}-k\right\|+\left(t-t_{0}\right)=r_{K}\left(y_{t}\right) .
\end{aligned}
$$

Thus, equality holds throughout these estimates, implying, in particular,

$$
\left\|y_{t_{0}}-k^{\prime}\right\|=\left\|y_{t_{0}}-k\right\|=r_{K}\left(y_{t_{0}}\right)
$$

Hence, $k^{\prime} \in Q_{K}\left(y_{t_{0}}\right)$. Finally, by the strict convexity of $X$, we obtain from (3.4) that $k^{\prime}=k$. Thus, if $t>t_{0}$, the ray points $y_{t}$ have the unique farthest point $k$.

To conclude the proof, it suffices to show that if $\left(x_{1}, k_{1}\right) \in Q_{K}$ such that $u=\frac{x_{1}-k_{1}}{r_{K}\left(x_{1}\right)}$ then $k_{1}=k$. Indeed, for some $t>t_{0}$ we have

$$
\begin{aligned}
r_{K}\left(y_{t}\right)+r_{K}\left(x_{1}\right) & =\left\|y_{t}-k+x_{1}-k_{1}\right\| \\
& \leqslant\left\|y_{t}-k_{1}\right\|+\left\|x_{1}-k\right\| \leqslant r_{K}\left(y_{t}\right)+r_{K}\left(x_{1}\right) .
\end{aligned}
$$

This yields $\left\|y_{t}-k_{1}\right\|=r_{K}\left(y_{t}\right)$, that is, $k_{1} \in Q_{K}\left(y_{t}\right)$. But $k$ is the unique farthest point of $y_{t}$. Hence, $k_{1}=k$.

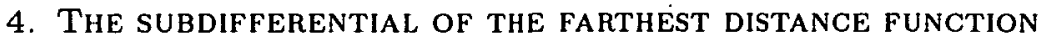

Let $K$ be a nonempty closed bounded subset of a Banach space $X$. It is well-known that the subdifferential $\partial r_{K}$ of the farthest distance function $r_{K}$ is a maximal monotone operator whose domain $D\left(\partial r_{K}\right)$ is all of $X$ and whose range $R\left(\partial r_{K}\right)$ is contained in the closed unit ball of $X^{*}$; moreover, if $X$ is reflexive, then $\overline{R\left(\partial r_{K}\right)}$ is a convex set. If, in addition, $X$ is a smooth space, we can even show that $R\left(\partial r_{K}\right)$ is dense in $\vec{B}^{*}(0 ; 1)$. For this we need the following simple lemma on the asymptotic behaviour of $\partial r_{K}(x)$ for $\|x\| \rightarrow \infty$. 
Lemma 4.1. Let $\left(\left(x_{n}, x_{n}^{*}\right)\right)_{n \in \mathbb{N}}$ be a sequence in $\partial r_{K}$ such that $\lim _{n \rightarrow \infty}\left\|x_{n}\right\|=\infty$. Then, for each $y \in X$,

$$
\lim _{n \rightarrow \infty}\left\langle\frac{x_{n}-y}{\left\|x_{n}-y\right\|}, x_{n}^{*}\right\rangle=\lim _{n \rightarrow \infty}\left\|x_{n}^{*}\right\|=1
$$

Thus, for each $\delta \in(0,1)$, the set $\left(\partial r_{K}\right)^{-1}\left(\bar{B}^{*}(0 ; 1-\delta)\right)$ is bounded.

Proof: If $y \in X$, then we have, for each $n \in \mathbb{N}$,

$$
\left\|x_{n}-y\right\| \leqslant r_{K}\left(x_{n}\right)+r_{K}(y)
$$

and

$$
r_{K}\left(x_{n}\right)+\left\langle y-x_{n}, x_{n}^{*}\right\rangle \leqslant r_{K}(y) .
$$

Combining these two inequalities and dividing them by $\left\|y-x_{n}\right\|$ yields

$$
1-\frac{2 r_{K}(y)}{\left\|x_{n}-y\right\|} \leqslant \frac{r_{K}\left(x_{n}\right)-r_{K}(y)}{\left\|x_{n}-y\right\|} \leqslant\left\langle\frac{x_{n}-y}{\left\|x_{n}-y\right\|}, x_{n}^{*}\right\rangle \leqslant\left\|x_{n}^{*}\right\| \leqslant 1,
$$

from which (4.1) follows as $n \rightarrow \infty$.

THEOREM 4.2. If $X$ is reflexive and smooth, then the range $R\left(\partial r_{K}\right)$ of $\partial r_{K}$ is a convex set satisfying

$$
B^{*}(0 ; 1) \subset R\left(\partial r_{K}\right) \text { and } \bar{B}^{*}(0 ; 1)=\overline{R\left(\partial r_{K}\right)}
$$

Proof: We first deduce the last assertion. By the convexity of $\overline{R\left(\partial r_{K}\right)}$ it suffices to show that $S^{*}\left(\begin{array}{ll}0 & 1\end{array}\right) \subset \overline{R\left(\partial r_{K}\right)}$.

Suppose $u^{*} \in S^{*}(0 ; 1)$ and $u \in S(0 ; 1)$ such that $u^{*} \in F(u)$. For each $n \in \mathbb{N}$, choose $x_{n}^{*} \in \partial r_{K}(n u)$. Then, by Lemma 4.1,

$$
\lim _{n \rightarrow \infty}\left\langle u, x_{n}^{*}\right\rangle=\lim _{n \rightarrow \infty}\left\|x_{n}^{*}\right\|=1
$$

The sequence $\left(x_{n}^{*}\right)$ contains a subsequence which is weakly convergent to a limit that belongs to the weakly closed set $\overline{R\left(\partial r_{K}\right)}$ as well as to $F(u)$. Since, by the smoothness of $X, F(u)$ is a singleton, this limit has to be the element $u^{*}$. Thus $u^{*} \in \overline{R\left(\partial r_{K}\right)}$.

Now, let $x^{*}$ be an element of the open unit ball $B^{*}(0 ; 1)$. By what we have just proven, there exists a sequence $\left(\left(x_{n}, x_{n}^{*}\right)\right)_{n \in \mathbb{N}}$ in $\partial r_{K}$ such that $\lim _{n \rightarrow \infty} x_{n}^{*}=x^{*}$. By Lemma 4.1 , the sequence $\left(x_{n}\right)$ is bounded in $X$, and thus has a weakly convergent subsequence with limit, say $x$. Since $\left(\partial r_{K}\right)^{-1}$ is demiclosed, we have $\left(x, x^{*}\right) \in \partial r_{K}$, which proves that $x^{*}$ belongs to the range of $\partial r_{K}$.

As $X^{*}$ is strictly convex, the relation $B^{*}(0 ; 1) \subset R\left(\partial r_{K}\right) \subset \bar{B}^{*}(0 ; 1)$ implies that $R\left(\partial r_{K}\right)$ is a convex set. 
To get more information on the inverse image of a single subgradient of $r_{K}$ we now consider the relation between the subdifferential $\partial r_{K}$ and the operator $\frac{I-Q_{K}}{r_{K}}$ of "ray directions".

Suppose from now on that $K$ contains at least two elements. Then the subdifferential $\partial r_{K}$ extends the monotone operator $F \cdot \frac{I-Q_{K}}{r_{K}}$. Indeed, if $(x, k) \in Q_{K}$ and $x^{*} \in$ $F\left(\frac{x-k}{r_{K}(x)}\right)$, then $\left\|x^{*}\right\|=1$ and $\left\langle x-k, x^{*}\right\rangle=r_{K}(x)$. Hence we have, for each $y \in X$,

$$
r_{K}(x)+\left\langle y-x, x^{*}\right\rangle=\left\langle y-k, x^{*}\right\rangle \leqslant\|y-k\|\left\|x^{*}\right\| \leqslant r_{K}(y) .
$$

Thus $x^{*} \in \partial r_{K}(x)$.

Since the farthest distance function of $K$ and $\overline{c o} K$ coincide, the above observations remain true if $Q_{K}$ is replaced by $Q_{\overline{c o} K}$. Furthermore, as $D\left(Q_{\overline{\mathrm{co}} K}\right)$ is dense in a reflexive space $X$, we obtain from Proposition 2.1:

Proposition 4.3. Suppose $X$ is reflexive. Then $\partial r_{K}$ is the unique maximal monotone extension of the operator $F \cdot \frac{I-Q_{\overline{c o} K}}{r_{K}}$, and for each $x \in X$,

$$
\partial r_{K}(x)=\bigcap_{\delta>0} \overline{\operatorname{co}}\left\{F\left(\frac{y-Q_{\overline{c o} K}(y)}{r_{K}(y)}\right) ; y \in B(x ; \delta)\right\}
$$

Furthermore, $\overline{R\left(\partial r_{K}\right)}=\overline{\mathrm{co}} R\left(F \cdot \frac{I-Q_{\overline{\mathrm{co}} K}}{r_{K}}\right)$.

If, in addition, the norm of $X^{*}$ is Fréchet differentiable, then the proposition holds true with $Q_{\overline{\mathrm{co}} K}$ replaced by $Q_{K}$.

As the range of $F \cdot \frac{I-Q_{K}}{r_{K}}$ and $F \cdot \frac{I-Q_{\overline{c o} K}}{r_{K}}$, respectively, is contained in the unit sphere of $X^{*}$, it seems reasonable to distinguish between those subgradients of $r_{K}$ which lie on the boundary of the unit ball of $X^{*}$ and those which are contained in its interior.

As a first result on subgradients lying on the unit sphere we have the following lemma, which extends the ray property of the farthest point mapping to the subdifferential of $r_{K}$.

Lemma 4.4. Suppose $u^{*} \in \partial r_{K}(x) \cap S^{*}(0 ; 1)$ for some $x \in X$, and let $u \in S(0 ; 1)$ be such that $u^{*} \in F(u)$. Then, for each positive number $t$,

$$
u^{*} \in \partial r_{K}(x+t u) \subset S^{*}(0 ; 1)
$$

Proof: Set $x_{t}:=x+t u$ for $t>0$. Then $\left\langle x_{t}-x, u^{*}\right\rangle=\left\|x_{t}-x\right\|=t$. Combining this with

$$
r_{K}(x)+\left\langle x_{t}-x, u^{*}\right\rangle \leqslant r_{K}\left(x_{t}\right) \leqslant r_{K}(x)+\left\|x_{t}-x\right\|
$$

gives $r_{K}\left(x_{t}\right)=r_{K}(x)+t$, whence $u^{*} \in \partial r_{K}\left(x_{t}\right)$ immediately follows. 
Now, let $x^{*} \in \partial r_{K}\left(x_{t}\right)$ for some $t>0$. Then the inequality

$$
0 \leqslant r_{K}(x)-r_{K}\left(x_{t}\right)-\left\langle x-x_{t}, x^{*}\right\rangle=-t+t\left\langle u, x^{*}\right\rangle \leqslant t\left(\left\|x^{*}\right\|-1\right) \leqslant 0
$$

implies that $\left\|x^{*}\right\|=1$.

Under suitable conditions on the underlying space all subgradients of $r_{K}$ that lie on the unit sphere belong to the range of the mapping $F \cdot \frac{I-Q_{K}}{r_{K}}$. In fact, if $X$ is reflexive and locally uniformly convex, then

$$
\partial r_{K}(x) \cap S^{*}(0 ; 1)=F\left(\frac{x-Q_{K}(x)}{r_{K}(x)}\right) \quad \forall x \in X .
$$

This formula can be obtained from Asplund [1], though it is not explicitly stated there. We shall prove a generalised version of (4.3) under different assumptions on the Banach space $X$.

Proposition 4.5. If $X$ is reflexive and has a Fréchet differentiable norm, then, for each $x \in X$,

$$
\partial r_{K}(x) \cap S^{*}(0 ; 1)=F\left(\frac{x-Q_{\overline{\mathrm{co}} K}(x)}{r_{K}(x)}\right) .
$$

If, in addition, also the norm of $X^{*}$ is Fréchet differentiable, then (4.4) holds with $Q_{\overline{c o} K}$ replaced by $Q_{K}$.

Proof: We give a proof that uses the representation (4.2) for $\partial r_{K}$.

If $x \in X$ and $u^{*} \in \partial r_{K}(x) \cap S^{*}(0 ; 1)$, let $u \in S(0 ; 1)$ be such that $u^{*}=F(u)$. For $n \in \mathbb{N}$ define

$$
U_{n}:=\left\{F\left(\frac{y-Q_{\overline{c o} K}(y)}{r_{K}(y)}\right) ; y \in B\left(x ; \frac{1}{n}\right)\right\} .
$$

Then $U_{n}$ has nonempty intersection with the half-space

$$
H_{n}:=\left\{x^{*} \in X^{*} ;\left\langle u, x^{*}\right\rangle>1-\frac{1}{n}\right\} .
$$

For, otherwise the complementary half-space $X^{*} \backslash H_{n}$ would contain the set $U_{n}$ and consequently also its closed convex hull, implying $u^{*} \in X^{*} \backslash H_{n}$, which contradicts $\left\langle u, u^{*}\right\rangle=1$. Hence, for each $n \in \mathbb{N}$, we can choose $u_{n}^{*} \in H_{n} \cap U_{n}$. Then $\lim _{n \rightarrow \infty}\left\langle u, u_{n}^{*}\right\rangle=1$. As the norm of $X$ is Fréchet differentiable at $u$ with derivative $u^{*}$, the sequence $\left(u_{n}^{*}\right)$ strongly converges to $u^{*}$. Moreover, since $u_{n}^{*} \in U_{n}$, there exists $\left(y_{n}, z_{n}\right) \in Q_{\overline{\mathrm{co}} K}$ such that $\left\|y_{n}-x\right\|<1 / n$ and $u_{n}^{*} \in F\left(\frac{y_{n}-z_{n}}{r_{K}\left(y_{n}\right)}\right)$. The sequence $\left(y_{n}\right)$ converges to $x$, and $\left(z_{n}\right)$ has a subsequence which is weakly convergent to a limit, say $z$, in $\overline{c o} K$. Hence, $\left\langle x-z, u^{*}\right\rangle=r_{K}(x)$ and $z \in Q_{\overline{c o} K}(x)$, which proves that

$$
u^{*} \in F\left(\frac{x-Q_{\overline{\bar{c}} K}(x)}{r_{K}(x)}\right) .
$$


If we pass to the inverse operators in (4.4), we obtain, under the assumptions of Proposition 4.5,

$$
\left(\partial r_{K}\right)^{-1}\left(u^{*}\right)=\left(\frac{I-Q_{\overline{\mathrm{co}} K}}{r_{K}}\right)^{-1} \cdot F^{-1}\left(u^{*}\right) \quad \forall u^{*} \in S^{*}(0 ; 1) .
$$

If, in addition, $X$ is strictly convex and $u^{*} \in R\left(\partial r_{K}\right) \cap S^{*}(0 ; 1)$, we can apply Proposition 3.1 to $F^{-1}\left(u^{*}\right) \in R\left(\frac{I-Q_{\overline{c o} K}}{r_{K}}\right)$. This shows that the set of elements having $u^{*}$ as a subgradient of $r_{K}$ is actually a ray. This result is stated in the first part of the following theorem. For convenience, we sharpen the assumptions on the space $X$ such that the farthest point mapping of the set $K$ itself, instead that of its closed convex hull, is involved. The second part of the theorem concerns those subgradients of $r_{K}$ which lie in the open unit ball of $X^{*}$.

THEOREM 4.6 .

(i) Suppose either $X$ is reflexive and locally uniformly convex or both $X$ and $X^{*}$ have a Fréchet differentiable norm. If $u^{*} \in R\left(\partial r_{K}\right) \cap S^{*}(0 ; 1)$, then there exists a unique element $k \in K$ such that $k \in Q_{K}\left(k+t F^{-1}\left(u^{*}\right)\right)$ for all sufficiently large $t$, and $\left(\partial r_{K}\right)^{-1}\left(u^{*}\right)$ is the ray

$$
\left(\partial r_{K}\right)^{-1}\left(u^{*}\right)=\left\{k+t F^{-1}\left(u^{*}\right) ; t \geqslant t_{0}\right\}
$$

where $t_{0}:=\min \left\{t>0 ; k \in Q_{K}\left(k+t F^{-1}\left(u^{*}\right)\right)\right\}$.

(ii) If $X$ is uniformly convex and smooth, then the restriction of the mapping $\left(\partial r_{K}\right)^{-1}$ to the open ball $B^{*}(0 ; 1)$ is single-valued and continuous with respect to the norm topologies of $X$ and $X^{*}$.

Proof: $B y$ the remarks preceding the theorem it is enough to prove the second part. Thus, let $x^{*} \in B^{*}(0 ; 1)$. By Theorem 4.2 , the set $\left(\partial r_{K}\right)^{-1}\left(x^{*}\right)$ is nonempty. If it were not a singleton, it would contain a line segment with endpoints, say $x_{0}$ and $x_{1}$. Set $x_{\alpha}:=(1-\alpha) x_{0}+\alpha x_{1}$ for $0 \leqslant \alpha \leqslant 1$. By the subgradient property, we obtain, for each $\alpha \in[0,1]$,

$$
r_{K}\left(x_{\alpha}\right)-r_{K}\left(x_{0}\right)=\left\langle x_{\alpha}-x_{0}, x^{*},\right\rangle
$$

whence

$$
r_{K}\left(x_{\alpha}\right)=(1-\alpha) r_{K}\left(x_{0}\right)+\alpha r_{K}\left(x_{1}\right)
$$

follows. Now choose $\alpha$ such that $(1-\alpha) r_{K}\left(x_{0}\right)=\alpha r_{K}\left(x_{1}\right)$, and let $\left(k_{n}\right)$ be a maximising sequence in $K$ for the corresponding $x_{\alpha}$, that is, $\lim _{n \rightarrow \infty}\left\|x_{\alpha}-k_{n}\right\|=r_{K}\left(x_{\alpha}\right)$. This implies

$$
\lim _{n \rightarrow \infty}\left\|\frac{x_{0}-k_{n}}{r_{K}\left(x_{0}\right)}+\frac{x_{1}-k_{n}}{r_{K}\left(x_{1}\right)}\right\|=2 \text {. }
$$


By the uniform convexity of $X$, it follows that

$$
\lim _{n \rightarrow \infty}\left(\frac{x_{0}-k_{n}}{r_{K}\left(x_{0}\right)}-\frac{x_{1}-k_{n}}{r_{K}\left(x_{1}\right)}\right)=0 .
$$

This shows that $x_{0}=x_{1}$, if $r_{K}\left(x_{0}\right)=r_{K}\left(x_{1}\right)$. Otherwise, (4.6) implies that the sequence $\left(k_{n}\right)$ strongly converges to a limit $k \in K$ and that

$$
\frac{x_{0}-k}{r_{K}\left(x_{0}\right)}=\frac{x_{1}-k}{r_{K}\left(x_{1}\right)}=: u
$$

where $u \in \bar{B}(0 ; 1)$. Then

$$
x_{1}-x_{0}=\left(r_{K}\left(x_{1}\right)-r_{K}\left(x_{0}\right)\right) u .
$$

Combining this with (4.5) for $\alpha=1$, we obtain $\left\langle u, x^{*}\right\rangle=1$, which contradicts the fact that $\left\|x^{*}\right\|<1$.

As for the continuity of $\left(\partial r_{K}\right)^{-1}$ on $B^{*}(0 ; 1)$, let $\left(x_{n}^{*}\right)$ be a sequence in $B^{*}(0 ; 1)$ which converges to a point $x^{*} \in B^{*}(0 ; 1)$. Set $x_{n}:=\left(\partial r_{K}\right)^{-1}\left(x_{n}^{*}\right)$ and $x:=\left(\partial r_{K}\right)^{-1}\left(x^{*}\right)$. As $\left(\partial r_{K}\right)^{-1}$ is maximal monotone, we know that the sequence $\left(x_{n}\right)$ weakly converges to $x$. We have to show that it is even strongly convergent. For each $n \in \mathbb{N}$ and each $\alpha \in[0,1]$, we have

$$
r_{K}(x)+\alpha\left\langle x_{n}-x, x^{*}\right\rangle \leqslant r_{K}\left((1-\alpha) x+\alpha x_{n}\right) \leqslant r_{K}(x)+\alpha\left\langle x_{n}-x, x_{n}^{*}\right\rangle .
$$

For $\alpha=1$ and $n \rightarrow \infty$ this implies that $\lim _{n \rightarrow \infty} r_{K}\left(x_{n}\right)=r_{K}(x)$. Now we proceed similarly as in the proof of the single-valuedness. For each $n \in \mathbb{N}$, we choose $\alpha_{n} \in[0,1]$ such that $\left(1-\alpha_{n}\right) r_{K}(x)=\alpha_{n} r_{K}\left(x_{n}\right)$. Then $\lim _{n \rightarrow \infty} \alpha_{n}=1 / 2$, and from (4.7) with $\alpha$ replaced by $\alpha_{n}$, we obtain, as $n \rightarrow \infty$,

$$
\lim _{n \rightarrow \infty} r_{K}\left(\left(1-\alpha_{n}\right) x+\alpha_{n} x_{n}\right)=r_{K}(x) .
$$

Hence, there exists a sequence $\left(k_{n}\right)$ in $K$ such that

$$
\lim _{n \rightarrow \infty}\left\|\left(1-\alpha_{n}\right) x+\alpha_{n} x_{n}-k_{n}\right\|=r_{K}(x)
$$

implying

$$
\lim _{n \rightarrow \infty}\left\|\frac{x-k_{n}}{r_{K}(x)}+\frac{x_{n}-k_{n}}{r_{K}\left(x_{n}\right)}\right\|=2,
$$

and thus $\lim _{n \rightarrow \infty} x_{n}=x$, by the uniform convexity of $X$. 


\section{The CONTINUITY SET $C_{K}$ AND ITS COMPLEMENT}

At the end of the previous section we described the inverse image of a single subgradient of $r_{K}$ depending on whether it belongs to the boundary or the interior of the unit ball of $X^{*}$. With this in mind one might consider the sets of elements of $X$ which have either at least one or all subgradients in the interior and the boundary of the unit ball of $X^{*}$, respectively. Among the four cases that can occur we are mainly interested in the set

$$
C_{K}:=\left\{x \in X ; \partial r_{K}(x) \subset S^{*}(0 ; 1)\right\}
$$

and its complement

$$
\left(\partial r_{K}\right)^{-1}\left(B^{*}(0 ; 1)\right)=\left\{x \in X ; \partial r_{K}(x) \cap B^{*}(0 ; 1) \neq \emptyset\right\} .
$$

Under suitable assumptions on the underlying space, $C_{K}$ is a set of continuity points, see below. In any case, $C_{K}$ contains the set of points at which $r_{K}$ is Fréchet differentiable (see [18]) and thus a dense $G_{\delta}$-set of $X$, if $X$ is reflexive. The set $C_{K}$ itself is then a dense $G_{\delta}$ of $X$. If, in addition, the norm of $X$ is Fréchet differentiable, then $C_{K}$ is exactly the set of Fréchet differentiability points of $r_{K}$ (see for example [19, 22]). Moreover, by Proposition 4.5, $C_{K}$ is contained in the domain of $Q_{\overline{c o} K}$ and

$$
\begin{aligned}
C_{K}=\{x \in X ; & F\left(\frac{x-Q_{\overline{\mathrm{co}} K}(x)}{r_{K}(x)}\right) \text { is a singleton and } \\
y & \left.\mapsto F\left(\frac{y-Q_{\overline{\mathrm{co}} K}(y)}{r_{K}(y)}\right) \text { is upper semicontinuous at } x\right\} .
\end{aligned}
$$

If, in addition, also the norm of $X^{*}$ is Fréchet differentiable, then

$$
C_{K}=\left\{x \in X ; Q_{K}(x) \text { is a singleton and } Q_{K} \text { is upper semicontinuous at } x\right\}
$$

see [18]. Thus, in this case, $C_{K}$ is the set of elements $x$ for which the optimisation problem to maximise the distance from $x$ within the set $K$ is well-posed in the sense that $x$ has a unique farthest point in $K$, which depends on $x$ continuously.

The set $C_{K}$ has a counterpart for nearest points, say $C_{K}^{\prime}$. Structural properties of the complement $X \backslash C_{K}^{\prime}$ have been studied by several authors under various assumptions on the space $X$ and by different methods. See for example Bartke-Berens [7], Balaganskî̂ [3, 4], Westphal-Frerking [25], Veselý [24], Konyagin [20], and the survey article of Balaganskiî-Vlasov [5]. Balaganskiî [3] also deals with farthest points. He shows that $X \backslash C_{K}$ is pathwise connected, if the norms of $X$ and $X^{*}$ are Fréchet differentiable. In the case that $X$ is uniformly convex and smooth, it follows from Theorem 4.6 (ii) that $X \backslash C_{K}$ is the continuous image of a convex set and thus pathwise connected. In the following theorem we prove that this connectedness property remains true in any reflexive Banach space. 
Theorem 5.1. If $X$ is reflexive, then $X \backslash C_{K}$ is pathwise connected.

Proof: For abbreviation, set $A:=\left(\partial r_{K}\right)^{-1}$ and $G:=B^{*}(0 ; 1)$. Let $x, y \in X \backslash C_{K}=$ $A(G)$ and $x^{*}, y^{*} \in G$ such that $\left(x^{*}, x\right),\left(y^{*}, y\right) \in A$.

Since $X$ is reflexive, we may pass to an equivalent Fréchet differentiable norm on $X$ which induces a Fréchet differentiable dual norm on $X^{*}$ (see [13], p. 160). This has the advantage that the resolvent $J_{\lambda}: X^{*} \rightarrow X^{*}$ and the Yosida approximation $A_{\lambda}: X^{*} \rightarrow X$ of $A$ (corresponding to the new norms) are single-valued and continuous with respect to the strong topologies of $X$ and $X^{*}$.

For each $\lambda>0$ define $p_{\lambda}:[0,1] \rightarrow X^{*}$ by

$$
p_{\lambda}(t):=(1-t)\left(x^{*}+\lambda F(x)\right)+t\left(y^{*}+\lambda F(y)\right) .
$$

Then the mapping $A_{\lambda} \cdot p_{\lambda}$ is a path in $X$ with initial point $x$ and endpoint $y$, and we shall see that if $\lambda>0$ is small enough, then $A_{\lambda} p_{\lambda}(t)$ belongs to the set $A(G)$ for each $t \in[0,1]$. Indeed, it is sufficient to take $\lambda$ such that $J_{\lambda} p_{\lambda}(t) \in G$, since $\left(J_{\lambda} p_{\lambda}(t), A_{\lambda} p_{\lambda}(t)\right) \in A$.

To obtain a suitable choice for $\lambda$, observe that the line segment

$$
P:=\left\{(1-t) x^{*}+t y^{*} ; t \in[0,1]\right\}
$$

is contained in the convex set $\overline{D(A)} \cap G$ and recall that by (2.1) for each $w^{*} \in \overline{D(A)}$, $J_{\lambda} v^{*}$ converges to $w^{*}$ if $\left(v^{*}, \lambda\right)$ tends to $\left(w^{*}, 0+\right)$. Since $G$ is an open neighbourhood of the compact set $P$, there are real numbers $\eta, \lambda_{0}>0$ such that $J_{\lambda} v^{*} \in G$ whenever $\operatorname{dist}\left(v^{*}, P\right)<\eta$ and $0<\lambda<\lambda_{0}$.

If we take $\lambda$ such that

$$
0<\lambda<\lambda_{0} \text { and } \lambda \cdot \max \{\|x\|,\|y\|\}<\eta,
$$

then we have, for each $t \in[0,1]$,

$$
\left\|p_{\lambda}(t)-\left((1-t) x^{*}+t y^{*}\right)\right\|=\lambda\|(1-t) F(x)+t F(y)\|<\eta,
$$

that is, $\operatorname{dist}\left(p_{\lambda}(t), P\right)<\eta$. Thus, $J_{\lambda} p_{\lambda}(t)$ is in $G$, as desired.

Concerning the set $G$ in the proof above, note that one only needs that $G$ is open and convex to conclude that the image $A(G)$ under a maximal monotone operator $A$ is pathwise connected. A slight modification of the proof yields this assertion even under the hypothesis that $G$ is open and $G \cap \overline{D(A)}$ is connected. This extends a theorem of Veselý [24] for Hilbert spaces to arbitrary reflexive Banach spaces.

By Theorem 5.1 it is clear that $X \backslash C_{K}$ is uncountable, if it contains at least two points. It may happen, however, that $X \backslash C_{K}$ is a singleton. Indeed, if the closed convex hull of $K$ is a closed ball, say, $\overline{c o} K=\bar{B}(x ; r)$ for some $x \in X$ and some $r \geqslant 0$, then, for each $y \in X, r_{K}(y)=r+\|y-x\|$, implying $\partial r_{K}(y)=F\left(\frac{y-x}{\|y-x\|}\right)$ and thus $y \in C_{K}$, if $y \neq x$, and $\partial r_{K}(x)=\bar{B}^{*}(0 ; 1)$, hence $x \in X \backslash C_{K}$. The following proposition shows that under appropriate assumptions on $X$ this case is the only exception. 
PROPOSITION 5.2. If $X$ is a reflexive, strictly convex space and its norm is Fréchet differentiable, then $X \backslash C_{K}$ is a singleton if and only if $\overline{c o} K$ is a closed ball.

PRoof: If $x \in X$ such that $X \backslash C_{K}=\{x\}$, then, by Theorem $4.2, \partial r_{K}(x)=\bar{B}^{*}(0 ; 1)$, implying $F\left(\frac{x-Q_{\overline{c o} K}}{r_{K}(x)}\right)=S^{*}(0 ; 1)$, by Proposition 4.5. As the duality mapping $F$ is bijective, the latter gives $Q_{\overline{\mathrm{co}} K}(x)=S\left(x ; r_{K}(x)\right)$, and hence $\overline{\mathrm{co}} K=\overparen{B}\left(x ; r_{K}(x)\right)$.

We conclude this section with some remarks on rays in the set $C_{K}$. If $u^{*}$ is a subgradient of $r_{K}$ of norm one, then by Lemma 4.4, for every $x \in\left(\partial r_{K}\right)^{-1}\left(u^{*}\right)$ and every $u \in F^{-1}\left(u^{*}\right)$, the ray $R:=\{x+t u ; t>0\}$ is contained in $C_{K} \cap\left(\partial r_{K}\right)^{-1}\left(u^{*}\right)$. Without loss of generality we assume that $\min \left\{t \in \mathbb{R} ; u^{*} \in \partial r_{K}(x+t u)\right\}$ is zero such that $\bar{R}=R \cup\{x\}$ is a maximal ray in $\left(\partial r_{K}\right)^{-1}\left(u^{*}\right)$. Then the question arises whether the initial point $x$ of $\bar{R}$ also belongs to $C_{K}$ or not. The following two examples show that both cases are possible. Let $X$ be the Euclidean plane and $K=\left\{\left(1-\varphi^{3}\right)(\cos \varphi, \sin \varphi) ; 0 \leqslant \varphi \leqslant 1\right\}$. Then $u^{*}=u:=(-1,0)$ generates the ray $R=\{(-t, 0) ; t>0\}$ in $C_{K}$ whose initial point $(0,0)$ also belongs to $C_{K}$. On the other hand, if $K$ is a closed ball with centre $x$ in an arbitrary Banach space $X$ and $u^{*}$ is any unit vector in $X^{*}$, then every $u \in F^{-1}\left(u^{*}\right)$ generates the ray $R=\{x+t u ; t>0\}$ and $x \in X \backslash C_{K}$.

\section{The farthest POINT MAPPING IN Hilbert SPACES}

If $X$ is a Hilbert space, then not only the operator $\frac{I-Q_{K}}{r_{K}}$ is monotone, but also $-Q_{K}$ itself. Indeed, if $\left(x_{1}, k_{1}\right),\left(x_{2}, k_{2}\right) \in Q_{K}$, then

$$
2\left\langle x_{1}-x_{2},-k_{1}+k_{2}\right\rangle=\left\|x_{1}-k_{1}\right\|^{2}-\left\|x_{1}-k_{2}\right\|^{2}+\left\|x_{2}-k_{2}\right\|^{2}-\left\|x_{2}-k_{1}\right\|^{2} \geqslant 0 .
$$

More generally, $-Q_{K}$ is cyclically monotone, that is, if $\left(x_{0}, k_{0}\right),\left(x_{1}, k_{1}\right), \ldots,\left(x_{n}, k_{n}\right) \in$ $Q_{K}$, then

$$
\sum_{j=0}^{n}\left\langle x_{j+1}-x_{j},-k_{j+1}\right\rangle \geqslant 0
$$

where $\left(x_{n+1}, k_{n+1}\right):=\left(x_{0}, k_{0}\right)$. The operator $-Q_{K}$ has a unique maximal monotone extension, which is described in the following theorem. For this and further information see also [23].

Theorem 6.1. If $X$ is a Hilbert space, then $-Q_{K}$ has a unique maximal monotone extension in $X \times X$, namely the subdifferential $\partial \psi_{K}$ of the continuous convex function $\psi_{K}: X \rightarrow \mathbb{R}$, defined by

$$
\psi_{K}(x):=\sup \left\{\frac{1}{2}\|k\|^{2}-\langle x, k\rangle ; k \in K\right\}=\frac{1}{2} r_{K}^{2}(x)-\frac{1}{2}\|x\|^{2} .
$$

The domain of $\partial \psi_{K}$ is the whole space $X$; its range satisfies $\overline{R\left(\partial \psi_{K}\right)}=-\overline{c o} K$. Moreover, for each $x \in X$,

$$
x+\partial \psi_{K}(x)=\partial\left(\frac{1}{2} r_{K}^{2}\right)(x)=r_{K}(x) \partial r_{K}(x)
$$


Proof: By definition, $\psi_{K}$ is the upper envelope of a family of affine functions and thus has to be convex.

If $(x, k) \in Q_{K}$, then $\psi_{K}(x)=\|k\|^{2} / 2-\langle x, k\rangle$, and, for each $y \in X$,

$$
\psi_{K}(x)+\langle y-x,-k\rangle=\frac{1}{2}\|k\|^{2}-\langle y, k\rangle \leqslant \psi_{K}(y)
$$

This shows that the subdifferential $\partial \psi_{K}$ is actually a maximal monotone extension of $-Q_{K}$. Its uniqueness follows from Proposition 2.1 , which also gives that $\overline{R\left(\partial \psi_{K}\right)}=$ $\overline{\mathrm{co}} R\left(-Q_{K}\right)$, the latter being equal to $-\overline{\mathrm{co}} K$, by (3.2).

The relation (6.1) between $\partial \psi_{K}, \partial\left(r_{K}^{2} / 2\right)$, and $\partial r_{K}$ is evident from the definition of $\psi_{K}$

The function $\psi_{K}$ is the counterpart of a function, say $\varphi_{K}$, which is related to nearest points and was introduced by Asplund [2] in 1969 for studying the convexity of Chebyshev sets in Hilbert spaces. Indeed, if $K$ is a closed subset of a Hilbert space, then $\varphi_{K}$ is defined by

$$
\varphi_{K}(x):=\sup \left\{\langle x, k\rangle-\frac{1}{2}\|k\|^{2} ; k \in K\right\}=\frac{1}{2}\|x\|^{2}-\frac{1}{2} d_{K}{ }^{2}(x),
$$

where $d_{K}(x):=\inf \{\|x-k\| ; k \in K\}$ is the usual distance function. The subdifferential $\partial \varphi_{K}$ is the unique maximal monotone extension of the nearest point mapping $P_{K}$. Moreover, as was observed by Berens-Westphal [9], $P_{K}$ is maximal monotone if and only if $K$ is convex.

Maximal monotony of $-Q_{K}$ for a bounded set $K$ is characterised by the next proposition, which is an immediate consequence of Minty's theorem.

Proposition 6.2. Suppose $X$ is a Hilbert space. Then $-Q_{K}$ is maximal monotone if and only if $K$ is a singleton.

The interplay between the two subdifferentials we are concerned with here allows us to sharpen and to extend some of the results that are true for non-Hilbert spaces. The following proposition is an improvement of Theorem 4.6 (ii).

Proposition 6.3. If $X$ is a Hilbert space, then, for each $\delta \in(0,1)$, the mapping $\left(\partial r_{K}\right)^{-1}$ satisfies a Lipschitz condition on $\bar{B}(0 ; 1-\delta)$. Thus any two points of $X \backslash C_{K}$ can be joined by a Lipschitz curve completely contained in $X \backslash C_{K}$.

Proof: For fixed $\delta \in(0,1)$, let

$$
M:=\sup \left\{r_{K}(x) ; x \in\left(\partial r_{K}\right)^{-1}(\bar{B}(0 ; 1-\delta))\right\}
$$

For $i=1,2$ choose $y_{i} \in \bar{B}(0 ; 1-\delta)$ and set $x_{i}:=\left(\partial r_{K}\right)^{-1}\left(y_{i}\right)$. By (6.1), we have $r_{K}\left(x_{i}\right) y_{i} \in x_{i}+\partial \psi_{K}\left(x_{i}\right)$, which implies $J\left(r_{K}\left(x_{i}\right) y_{i}\right)=x_{i}$, where $J$ denotes the resolvent $\left(I+\partial \psi_{K}\right)^{-1}$ of $\partial \psi_{K}$. By the contraction property of $J$ as well as of $r_{K}$, we obtain

$$
\left\|x_{1}-x_{2}\right\|=\left\|J\left(r_{K}\left(x_{1}\right) y_{1}\right)-J\left(r_{K}\left(x_{2}\right) y_{2}\right)\right\|
$$




$$
\begin{aligned}
& \leqslant\left\|r_{K}\left(x_{1}\right) y_{1}-r_{K}\left(x_{2}\right) y_{2}\right\| \\
& \leqslant r_{K}\left(x_{1}\right)\left\|y_{1}-y_{2}\right\|+\left\|y_{2}\right\|\left\|x_{1}-x_{2}\right\| \\
& \leqslant M\left\|y_{1}-y_{2}\right\|+(1-\delta)\left\|x_{1}-x_{2}\right\|
\end{aligned}
$$

from which

$$
\left\|x_{1}-x_{2}\right\| \leqslant \frac{M}{\delta}\left\|y_{1}-y_{2}\right\|
$$

is deduced.

The next theorem describes the resolvent $\left(I+\partial \psi_{K}\right)^{-1}$ on rays starting at the origin.

ThEOREM 6.4. Suppose $X$ is a Hilbert space and $|K| \geqslant 2$. Let $J$ denote the resolvent $\left(I+\partial \psi_{K}\right)^{-1}$ of $\partial \psi_{K}$. Then, for each $u \in S(0 ; 1)$, the mapping

$$
[0, \infty) \ni t \mapsto J(t u)
$$

is a Lipschitz curve which starts at the Chebyshev centre of $K$, that is, the unique point in $X$ at which $r_{K}$ attains its infimum, and runs to infinity.

If $u \notin R\left(\partial r_{K}\right)$, then the curve is completely contained in the set $X \backslash C_{K}$.

If $u \in R\left(\partial r_{K}\right)$, then there is a positive number $t_{0}$ such that

$$
J(t u) \in \begin{cases}X \backslash C_{K} & \text { if } 0 \leqslant t<t_{0} \\ C_{K} & \text { if } t>t_{0} .\end{cases}
$$

Proof: For $t \geqslant 0$ set $x_{t}:=J(t u)$. Then by $(6.1)$,

$$
t u \in r_{K}\left(x_{t}\right) \partial r_{K}\left(x_{t}\right)
$$

If $t=0$, this gives $0 \in \partial r_{K}\left(x_{0}\right)$ and hence $x_{0} \in X \backslash C_{K}$. If $x_{t} \in C_{K}$ for some $t>0$, then (6.2) implies

$$
r_{K}\left(x_{t}\right)=t \quad \text { and } \quad u \in \partial r_{K}\left(x_{t}\right)
$$

Thus $x_{t} \in X \backslash C_{K}$ for each $t \geqslant 0$, if $u \notin R\left(\partial r_{K}\right)$.

On the other hand, if $u \in R\left(\partial r_{K}\right)$, then by Theorem 4.6 (i) there is a point $k \in K$ such that $\left(\partial r_{K}\right)^{-1}(u)=\left\{k+t u ; t \geqslant t_{0}\right\}$ where $t_{0}=\min \left\{t>0 ; k \in Q_{K}(k+t u)\right\}$. Then for each $t \geqslant t_{0}$, we have $r_{K}(k+t u)=t$, and by (6.1), tu $\in\left(I+\partial \psi_{K}\right)(k+t u)$ implying $x_{t}=k+t u$. By Lemma 4.4, $x_{t} \in C_{K}$ for each $t>t_{0}$. If $x_{t}$ belonged to $C_{K}$ also for some $t \in\left(0, t_{0}\right)$, then (6.3) would hold for this $t$, and by the representation of $\left(\partial r_{K}\right)^{-1}(u)$ as a ray, $x_{t}=k+t_{1} u$ for some $t_{1} \geqslant t_{0}$. As $r_{K}\left(x_{t}\right)=t$ and $r_{K}\left(k+t_{1} u\right)=t_{1}$, we have $t=t_{1}$, which is a contradiction. Hence, $x_{t} \in X \backslash C_{K}$ for each $t \in\left[0, t_{0}\right)$.

Note that the pathwise connectedness of the set $X \backslash C_{K}$ in case $X$ is a Hilbert space can be deduced also from Theorem 6.4. 


\section{REFERENCES}

[1] E. Asplund, 'Farthest points in reflexive locally uniformly rotund Banach spaces', Israel J. Math. 4 (1966), 213-216.

[2] E. Asplund, 'Chebyshev Sets in Hilbert Space', Trans. Amer. Math. Soc. 144 (1969), 235-240.

[3] V. S. Balaganskiī, 'On the connection between approximation and geometric properties of sets', (Russian), in Approximation in concrete and abstract Banach spaces, Akad. Nauk SSSR (Urals'kiǐ Naučnyi Centr, Sverdlovsk, 1987), pp. 46-53.

[4] V.S. Balaganskiǐ, 'On the connectedness of the set of points of discontinuity of the metric projection', East J. Approx. 2 (1996), 263-279.

[5] V.S. Balaganskii and L. P. Vlasov, 'The problem of convexity of Chebyshev sets', Russian Math. Surveys 51 (1996), 1127-1192.

[6] V. Barbu, Nonlinear semigroups and differential equations in Banach spaces (Noordhoff International Publishing, Leyden, 1976).

[7] K. Bartke and H. Berens, 'Eine Beschreibung der Nichteindeutigkeitsmenge für die beste Approximation in der euklidischen Ebene', J. Approx. Theory 47 (1986), 54-74.

[8] H. Berens, 'Best approximation in Hilbert space', in Approximation theory III, (E.W. Cheney, Editor) (Academic Press, New York, 1980), pp. 1-20.

[9] H. Berens and U. Westphal, 'Kodissipative metrische Projektionen in normierten linearen Räumen', in Linear spaces and approximation, (P. L. Butzer and B. Sz.-Nagy, Editors) (Birkhäuser, Basel, 1978), pp. 120-130.

[10] J. Blatter, 'Weiteste Punkte und nächste Punkte', Rev. Roumaine Math. Pures Appl. 14 (1969), 615-621.

[11] H. Brézis, Opérateurs maximaux monotones et semi-groupes de contractions dans les espaces de Hilbert, Mathematics Studies 5 (North-Holland Publishing Company, Amsterdam, 1973).

[12] I. Cioranescu, Geometry of Banach spaces, duality mappings and nonlinear problems, Mathematics and its Applications 62 (Kluwer, Dordrecht, Boston, London, 1990).

[13] M. M. Day, Normed linear spaces, (3rd ed.) (Springer-Verlag, Berlin, Heidelberg, New York, 1973).

[14] K. Deimling, Nonlinear functional analysis (Springer-Verlag, Berlin, Heidelberg, New York, 1985).

[15] R. Deville and V. Zizler, 'Farthest points in $w^{*}$-compact sets', Bull. Austral. Math. Soc. 38 (1988), 433-439.

[16] M. Edelstein, 'Farthest points in uniformly convex Banach spaces', Israel J. Math. 4 (1966), 171-176.

[17] M. Edelstein and J. Lewis, 'On exposed and farthest points in normed linear spaces', J. Austral. Math. Soc. 12 (1971), 301-308.

[18] S. Fitzpatrick, 'Metric projections and the differentiability of distance functions', Bull. Austral. Math. Soc. 22 (1980), 291-312.

[19] J. R. Giles, Convex analysis with application in the differentiation of convex functions (Pitman, Boston, London, Melbourne, 1982).

[20] S. V. Konyagin, 'Set of points of discontinuity of a metric projection on Chebyshev sets in Hilbert space', in Internat. Conf. on Theory of Approximation, Kaluga 1996, Abstracts of lectures 1, (1996), pp. 120-121. 
[21] K.-S. Lau, 'Farthest points in weakly compact sets', Israel J. Math. 22 (1975), 168-174.

[22] R. R. Phelps, Convex functions, monotone operators and differentiability, Lecture Notes in Math. 1364 (Springer-Verlag, Berlin, Heidelberg, New York, 1989).

[23] T. Schwartz, 'Farthest points and monotonicity methods in Hilbert spaces', in Approximation and optimization I, (D. D. Stancu et al., Editors) (Transilvania Press, Cluj-Napoca, 1997), pp. 351-356.

[24] L. Veselý, 'A connectedness property of maximal monotone operators and its application to approximation theory', Proc. Amer. Math. Soc. 115 (1992), 663-667.

[25] U. Westphal and J. Frerking, 'On a property of metric projections onto closed subsets of Hilbert spaces', Proc. Amer. Math. Soc. 105 (1989), 644-651.

[26] E. Zeidler, Nonlinear functional analysis and its applications II/B, Nonlinear Monotone Operators (Springer-Verlag, Berlin, Heidelberg, New York, 1990).

[27] N. V. Zhivkov, 'Continuity and non-multivaluedness properties of metric projections and antiprojections', Serdica 8 (1982), 378-385.

[28] N. V. Zhivkov, 'Compacta with dense ambiguous loci of metric projections and antiprojections', Proc. Amer. Math. Soc. 123 (1995), 3403-3411.

Institut für Mathematik

Universität Hannover

Welfengarten 1

30167 Hannover

Germany

e-mail: westphal@math.uni-hannover.de schwartz@math.uni-hannover.de 